\title{
AEROSPACE SYSTEMS DESIGN IN NASA'S COLLABORATIVE ENGINEERING ENVIRONMENT
}

Donald W. Monell and William M. Piland

NASA Langley Research Center

Hampton, Virginia 23681-2199

50th International Astronautical Congress

October 4-8, 1999

Amsterdam, The Netherlands 


\title{
Aerospace Systems Design in NASA's Collaborative Engineering Environment
}

\author{
Donald W. Monell* and William M. Piland** \\ NASA Langley Research Center \\ Hampton, Virginia 23681-2191
}

\begin{abstract}
Past designs of complex aerospace systems involved an environment consisting of collocated design teams with project managers, technical discipline experts, and other experts (e.g., manufacturing and systems operations). These experts were generally qualified only on the basis of past design experience and typically had access to a limited set of integrated analysis tools. These environments provided less than desirable design fidelity, often led to the inability of assessing critical programmatic and technical issues (e.g., cost, risk, technical impacts), and generally derived a design that was not necessarily optimized across the entire system. The continually changing, modern aerospace industry demands systems design processes that involve the best talent available (no matter where it resides) and access to the best design and analysis tools.
\end{abstract}

A solution to these demands involves a design environment referred to as collaborative engineering. The collaborative engineering environment evolving within the National Aeronautics and Space Administration (NASA) is a capability that enables the Agency's engineering infrastructure to interact and use the best state-of-the-art tools and data across organizational boundaries. Using collaborative engineering, the collocated team is replaced with an interactive team structure where the team members are geographically distributed and the best engineering talent can be applied to the design effort regardless of physical location. In addition, a more efficient, higher quality design product is delivered by bringing together the best engineering talent with more up-to-date design and analysis tools. These tools are focused on interactive, multidisciplinary design and analysis with emphasis on the complete life cycle of the system, and they include nontraditional, integrated tools for life cycle cost estimation and risk assessment.

NASA has made substantial progress during the last two years in developing a collaborative engineering environment. NASA is planning to use this collaborative engineering infrastructure to provide better aerospace systems life cycle design and analysis, which includes analytical assessment of the technical and programmatic aspects of a system from "cradle to grave."

This paper describes the recent NASA developments in the area of collaborative engineering, the benefits (realized and anticipated) of using the developed capability, and the long-term plans for implementing this capability across the Agency.

\section{INTRODUCTION}

Over the past several years, many government and industrial aerospace organizations have undergone workforce and budgets cutbacks. Many of these cutbacks have involved significant reductions in highly skilled technical personnel. Further, in many instances these reductions may have been severe enough to raise concerns for the ability of the organization to accomplish its mission or to accomplish it with sufficient quality to remain viable in the increasingly competitive aerospace environment. NASA has undergone about a $25 \%$ reduction in its workforce within the past 6 to 7 years. In the same time period, the Agency has focused its technical capabilities in a way that distributes the primary technical competency

* Deputy Element Manager, CEE, ISE

**Director, Independent Program Assessment Office

This paper is declared a work of the U.S. Government and is subject to copyright protection in the United States. 
areas across the Agency. Technical "Lead Centers" have been established to serve NASA's engineering requirements for the future. As a result, some Centers now find they are in the difficult position of often having to rely on the contributions of one or more other Centers if a quality technical aerospace systems design is to be developed. Inter-Center and inter-organizational design teams are being established on a regular basis to deliver the best design effort with the best engineering talent and tools available.

Recognizing that it is impractical for NASA's design and analysis teams to be colocated for the time it may take to complete a design activity, the NASA has begun an investment in the capability for geographically distributed team members to work together as efficiently as if they were physically together. The capability being created is called the Collaborative Engineering Environment (CEE). Although NASA still has much to do to apply the technologies needed for collaborative design efforts, the Agency is rapidly assembling the capability with high payoff expectations. The following discussion describes the $\mathrm{CEE}$, the status and plans for its development and future upgrade, and the benefits already derived from the capability.

\section{BACKGROUND}

About two years ago, NASA identified the need for an advanced aerospace systems concept design and life cycle analysis capability for Agency-wide use. The need for this capability first led to a request from the Office of the Administrator to the NASA Langley Research Center to organize and lead the deployment of an Agency "core" collaborative engineering capability by the end of Fiscal Year 1998. Secondly, in March 1998, the NASA Administrator charged the NASA Engineering Management Council (EMC) with the development and implementation of a "Future Tools" Program that would focus on developing the next generation of design and analysis tools and establish NASA as a world leader in engineering design. In response to the Administrator's charge, the NASA Chief Engineer and the
NASA Chief Technologist requested Langley to formulate an Agency-wide plan for the development and implementation of the activities associated with the "Future Tools" Program. As a result of these actions, the current Langley Research Center plan consists of establishing a Collaborative Engineering Environment (CEE) using analysis tools currently available and a farther-term activity to develop integrated, multidisciplinary systems design and life cycle analysis tools that advance the current state-ofthe-art in design capability. The combined program that the NASA Administrator requested is now called the Intelligent Synthesis Environment (ISE) Program. Langley formed an interCenter team, consisting of representatives from across NASA, to begin defining these two aspects of the ISE Program. In June 1998, the team produced a program plan for the implementation of the CEE, which led to the initial deployment of a core capability across the Agency.

\section{COLLABORATIVE ENGINEERING CONCEPT}

One solution to the needs and demands of design and analysis engineers working together with acceptable tools, but not colocated, involves the development of an aerospace design environment referred to as the Collaborative Engineering Environment (CEE). Using collaborative engineering, the team becomes an interactive team structure through electronic means, and the best engineering talent can be applied to the design effort regardless of physical location. Bringing together the best engineering talent with state-ofbest-practice design and analysis tools produces a more efficient, higher quality design product. The tools incorporated in the CEE focus on interactive, multidisciplinary design and analysis with emphasis on the complete life cycle of the aerospace system. They also include nontraditional, integrated tools for life cycle cost estimation and risk assessment. Thus, the CEE consists of three primary elements: (1) team members; (2) analysis hardware and software; and, (3) data (Figure 1). 


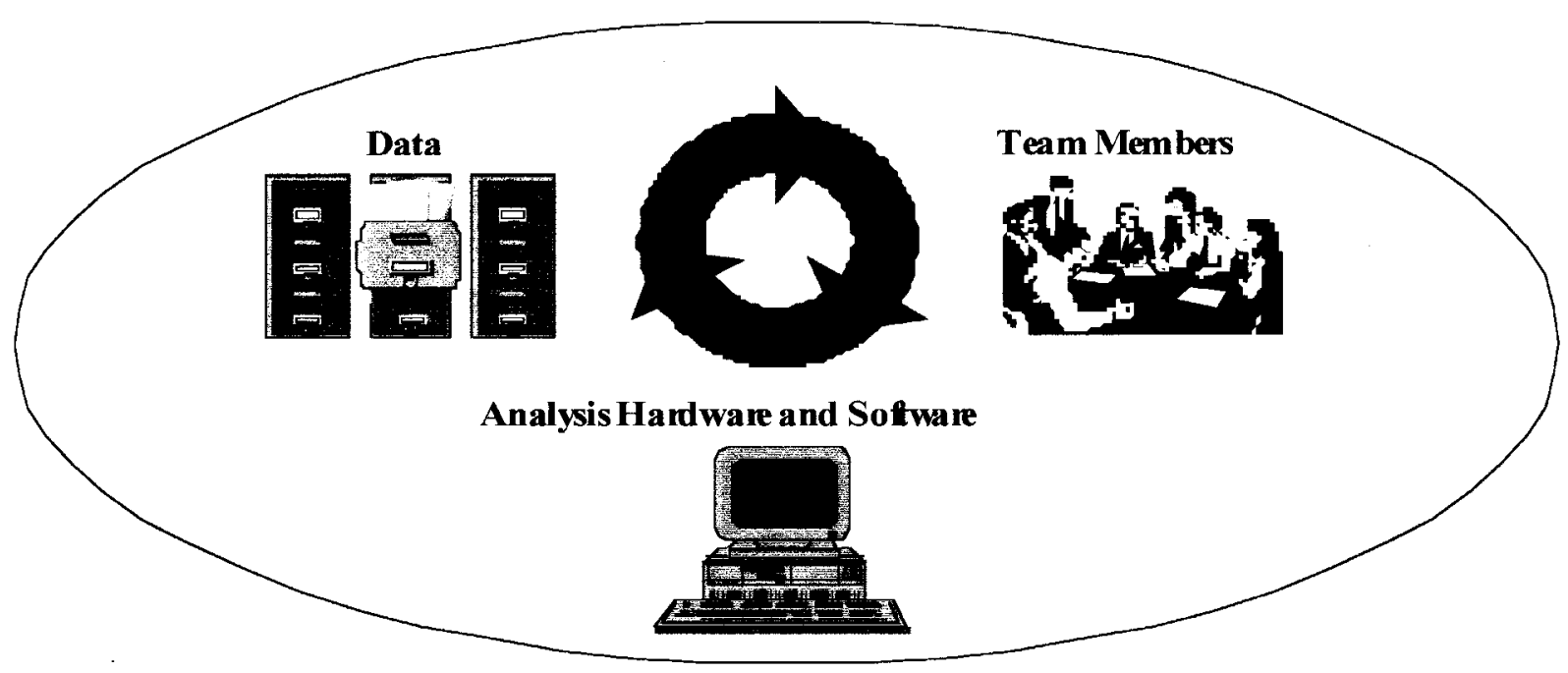

Figure 1 - Elements of Collaboration

\section{REQUIREMENTS}

The Collaborative Engineering Environment enables geographically distributed experts to interact and contribute to a project. The first step towards establishing the environment was to develop the requirements that would guide the definition, design, and deployment of the capabilities. The top-level requirements that were identified by the CEE team were:

- provide synchronous (real-time) and asynchronous (not real-time) collaboration of geographically distributed analysis team members

- develop/establish an operating environment that ties individual analysis tools (technical, cost, schedule, and risk) together in an interactive manner, and allows each NASA Center to use them to perform life cycle analyses

- provide a necessary level of security for all project design and analysis data

- provide for incorporation of future ISE advanced computing, communications, and analysis technologies into the CEE architecture
Some of the additional, secondary requirements that were used to derive the infrastructure design are listed below:

- enable remote users to receive design/analysis information

- enable remote users to upload design/analysis evaluation

- enable all users to interact with each others data at any time

- enable all users to communicate and interact with each other in real time

- capture the design and analysis process electronically for report generation and database development

- allow some degree of device independence for remote users so that they can quickly and easily be brought into the $\mathrm{CEE}$

The CEE is evolving to a capability that enables the agency's engineering infrastructure to interact across organizational boundaries and use the best state-of-the-art tools and data (See Figure 2). NASA is planning to use this collaborative engineering infrastructure to conduct better aerospace systems design and analysis, and to provide analytical assessment of the technical and programmatic aspects of a system over its complete life cycle. 


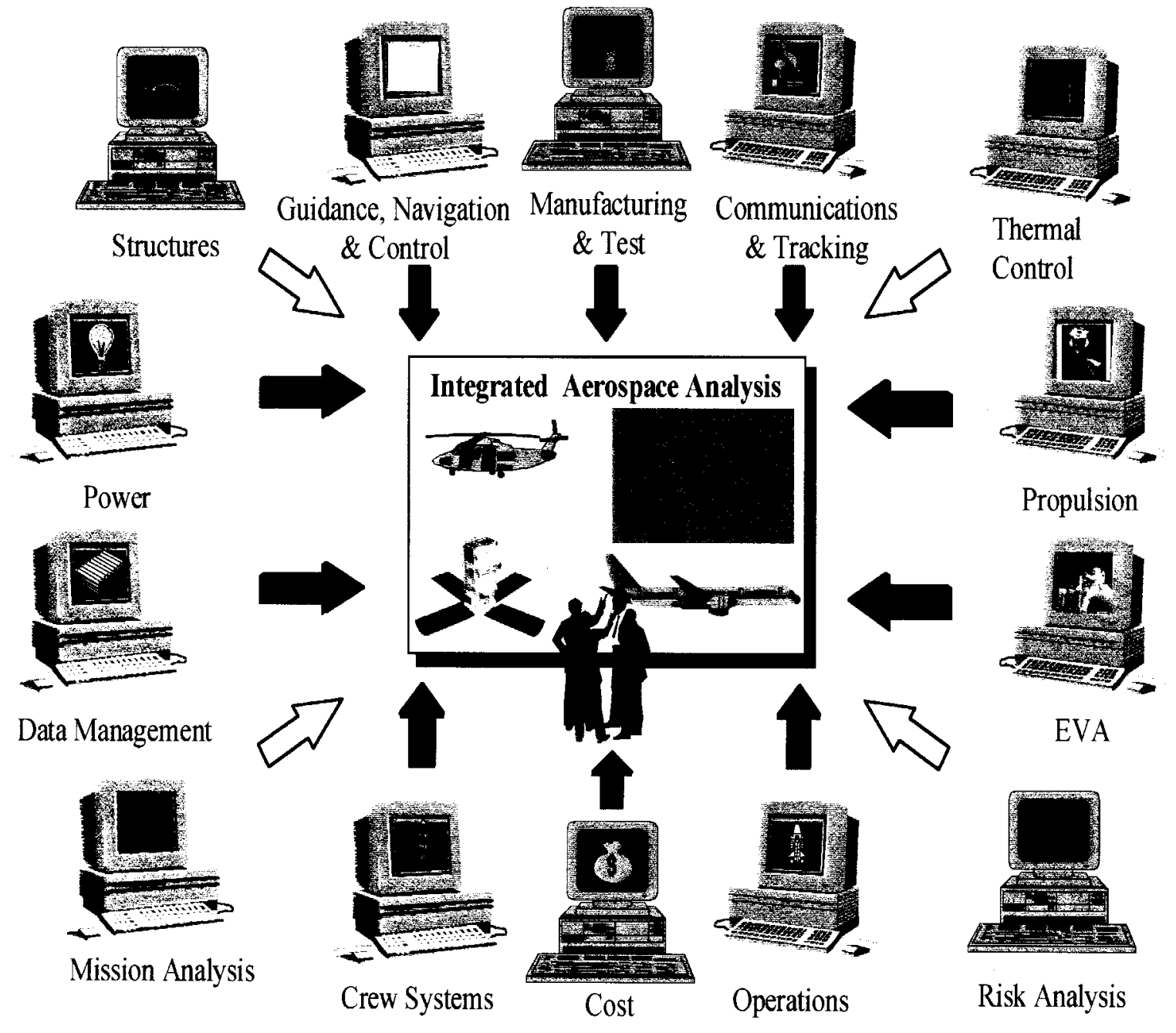

Figure 2 - Integration \& Linking of Expertise

\section{TEAM MEMBERS}

Integration of a group of people working toward a common goal has previously been accomplished using Integrated Product Development Teams (IPDT). For tasks involving systems design and analysis, an IPDT was typically a group of all personnel necessary to accomplish an aerospace system design (e.g., program analysts, engineers, technicians, cost analysts, etc.), and these people were typically colocated for the duration of the design phase. The primary negative aspect of the IPDT approach was that it normally required individuals to relocate to a single site, frequently removing them from their "home" environment, tools, and expert material. The approach that NASA is taking will be to provide services that enable collaboration among the team members from their desks or any one of the Collaborative Engineering Centers being established across the Agency (Figure 3). The services that are being employed are: 


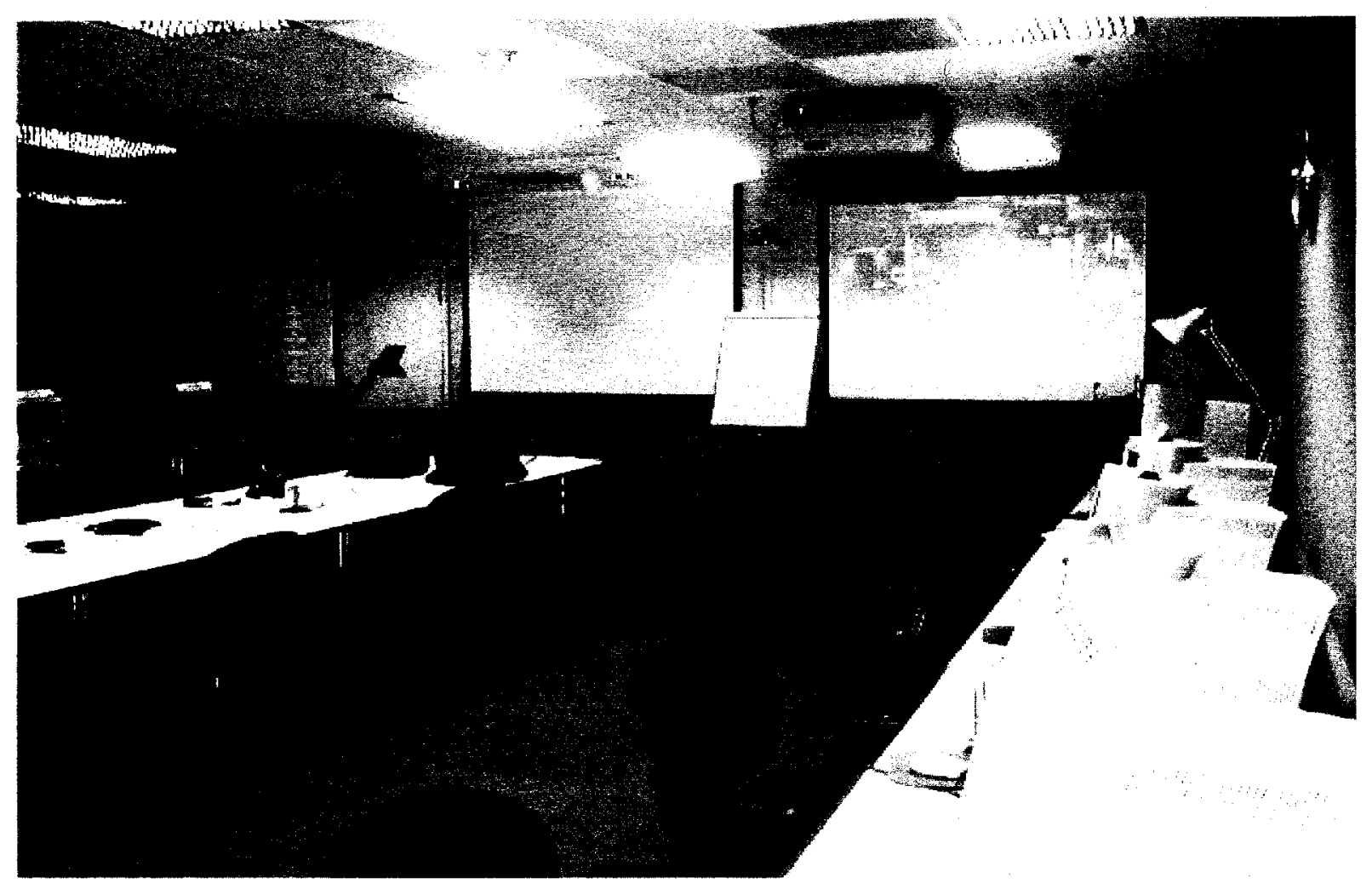

Figure 3 - Collaborative Engineering Center

- A Collaborative Knowledge Management tool (currently Opentext's Livelink ${ }^{\mathrm{TM}}$ ) - enabling workflow management, task tracking, centralized team communications, and data repository

- A Video Conferencing Capability (currently using Picturetel ${ }^{\mathrm{TM}}$ equipment)

- An Application Sharing Capability (currently using Microsoft's NetMeeting ${ }^{\mathrm{TM}}$ ) - enabling real-time document and data sharing during teleconferences or videoconferences

- Projection and Video display capabilities

- Numerous hardware computing platforms

NASA is already utilizing these new collaborative teaming arrangements to accomplish large and small project reviews, training, and some limited design and development activities. All three of these areas of use will be expanded in the near future. In particular, the Revolutionize Cultural Change, Training and Education (RCCTE) element of ISE is planning extensive use of these rooms to train a broad spectrum of NASA engineers and managers on the use of collaborative technologies.

To accomplish the efficient use of these CEE Centers, NASA has identified an individual at each NASA Center with responsiblity for coordinating the use of the room. That individual is fully qualified to setup each of the services available (i.e., video-conferencing, data sharing, etc.) and perform system-level testing when problems arise.

\section{ANALYSIS SOFTWARE AND HARDWARE}

The initial deployment of the core CEE capability at all NASA Centers consists of installation of the following hardware:

- Large Room Video Conferencing System enables multi-Center videoconferencing 
- Ceiling Mounted 1280x1024 LCD Projector and Large Display Screen - enables display of presentations and analysis results to design team in a CEE Center

- Document Conferencing System - enables presentation/display of "hardcopy" documents to geographically dispersed design team

- Presentation Display PC (Pentium II class with DVD drive) - enables management of presentation materials

- System Modeling PC (Pentium II class) enables high-level system or subsystem analysis to be performed in a collaborative setting

- Unix Workstation - enables low-level (detailed) system or subsystem analysis to be performed in a collaborative setting

- TV, VCRs and 1280x1024 Scan Converter enables introduction of data in video format into collaborative team sessions

- Teleconference Phone

- Video Switch Box and Interface Box enables control of video source displayed to collaborative team

- Remote Control System

As NASA plans and implements the Collaborative Engineering Environment, the intent will be to ensure a level of commonality, or standardization, across all CEE Centers. In order to accomplish this, the CEE element of the ISE Program has established an Infrastructure Development Team. This team is coordinating the definition, acquisition, and deployment of the hardware for all of the Centers. The team is working very closely with other NASA information technology development organizations to enhance the CEE capabilities and ensure that the CEE hardware and services do not interfere with other computer resources at any NASA Center.

The CEE goal is to incorporate design and analysis tool integration approaches that enable:
(1) the seamless integration of analysis capabilities within a preliminary-level or detailedlevel design phase; and, (2) the ability to analyze at increasingly complex levels of detail within a discipline (i.e., throughout the design phases). Figure 4 is a notional depiction of this goal. As illustrated, there are a number of levels of design and analysis that can be incorporated into a system design. For example, these levels range from a system concept design (or preliminary design) level to a design level with sufficient maturity that defines all the final specifications required for system construction. The particular discipline tools associated with each design level represent the degree of sophistication, or rigor, needed to support a system design at that level. The CEE's current task involves assembling the available and applicable tools, organizing them according to level of design level application, and integrating the tools to work together to achieve a system design at the desired level of maturity. As further illustrated, there are gaps in the availability of many discipline tools at certain design levels. A major goal of the ISE Program is to fill these gaps by developing the appropriate discipline design and analysis tools, complete the multidisciplinary integration, infuse the advanced tool set into the CEE capability, and thus improve NASA's ability to efficiently perform its aerospace systems development activities. A team of NASA experts with support from industry and academia partners is beginning to assess the required functionality of the environment that will support the integration of the design and analysis tools. Numerous integration methods and techniques are being investigated to determine the best approach. The near-term solution to tool integration may involve using the Common Object Request Broker Architecture (CORBA). CORBA allows software tools to interact with each other by creating a common interface methodology. However, the far-term goal is to link tools via "smart interfaces" that will enable a "plug-andplay"-type integration. 


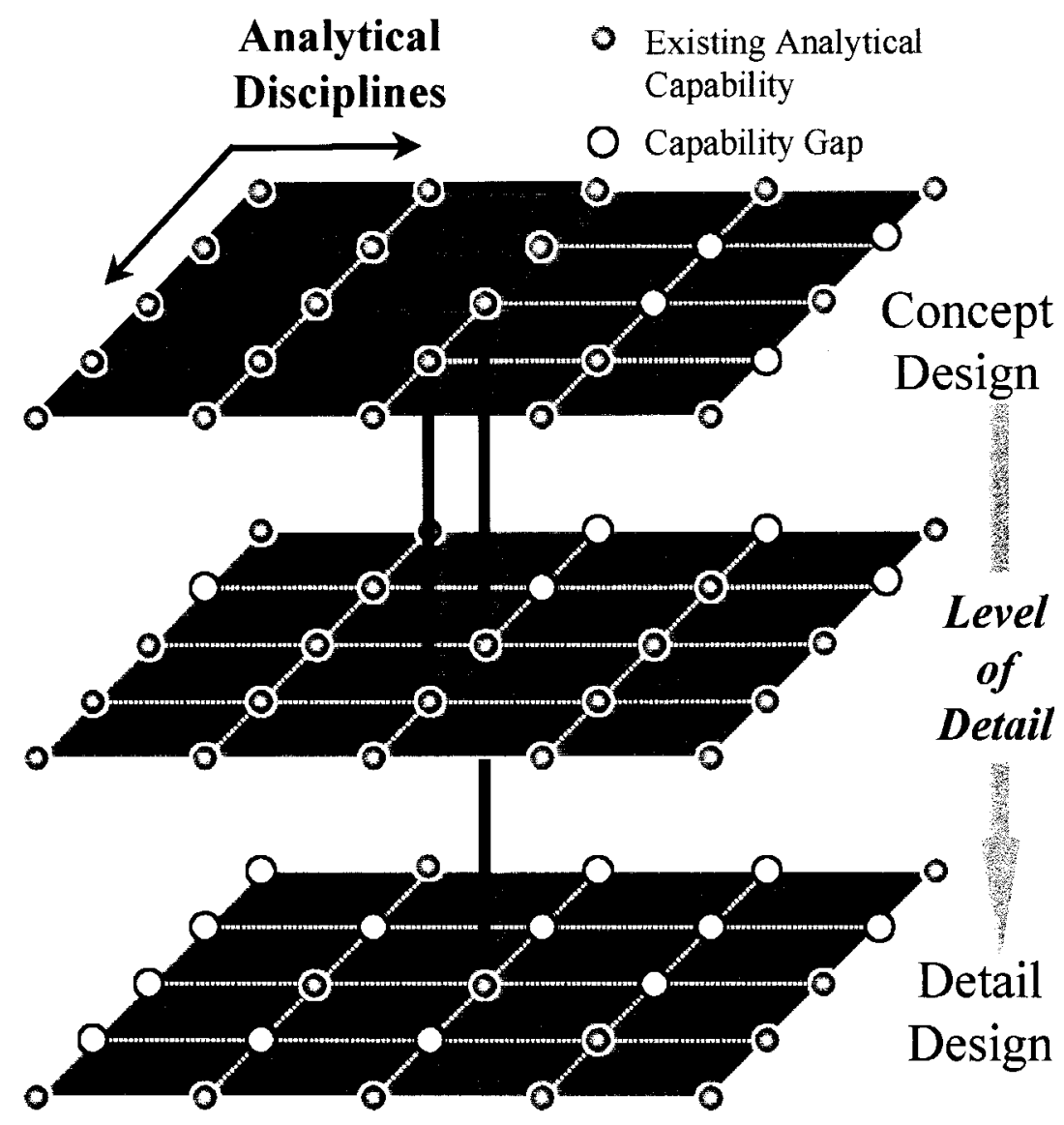

Figure 4 - Integrated Analysis

\section{$\underline{\text { DATA }}$}

The ability to share data between personnel and across geographically distributed organizations is absolutely critical to the success of all future NASA missions. The approach for data sharing that the CEE will use is a Virtual Private Network (VPN) that allows collaboration with sufficient security provisions to protect each NASA Center's computer resources and the CEE design and analysis data. The VPN consists of "ISE/CEE Access Nodes" (ICAN), at each Center, connected via one of the NASA networks (see Figure 5). Personnel requiring the services of the CEE infrastructure (e.g., shared file system, NET Meeting, etc.) will access the local ICAN for security authentication. Once authentication has been received, the individual can access any of the other ICANs across NASA and utilize the services provided. Security is also maintained by the firewall between an organization's other computing resources and the ICAN. NASA is currently developing agreements among several organizations in the Agency (e.g., Chief Information Officer's (CIO) Agency team, the NASA Integrated Services Network (NISN) organization, and the NASA Research and Education Network (NREN), etc.). These agreements will ensure that all of the elements of the CEE infrastructure will be in place and available for use by the CEE. NASA is also partnering with other U. S. Government Agencies to design, test, and develop this capability. In the proposed approach, security is the primary concern, and strict procedures will be enforced. 


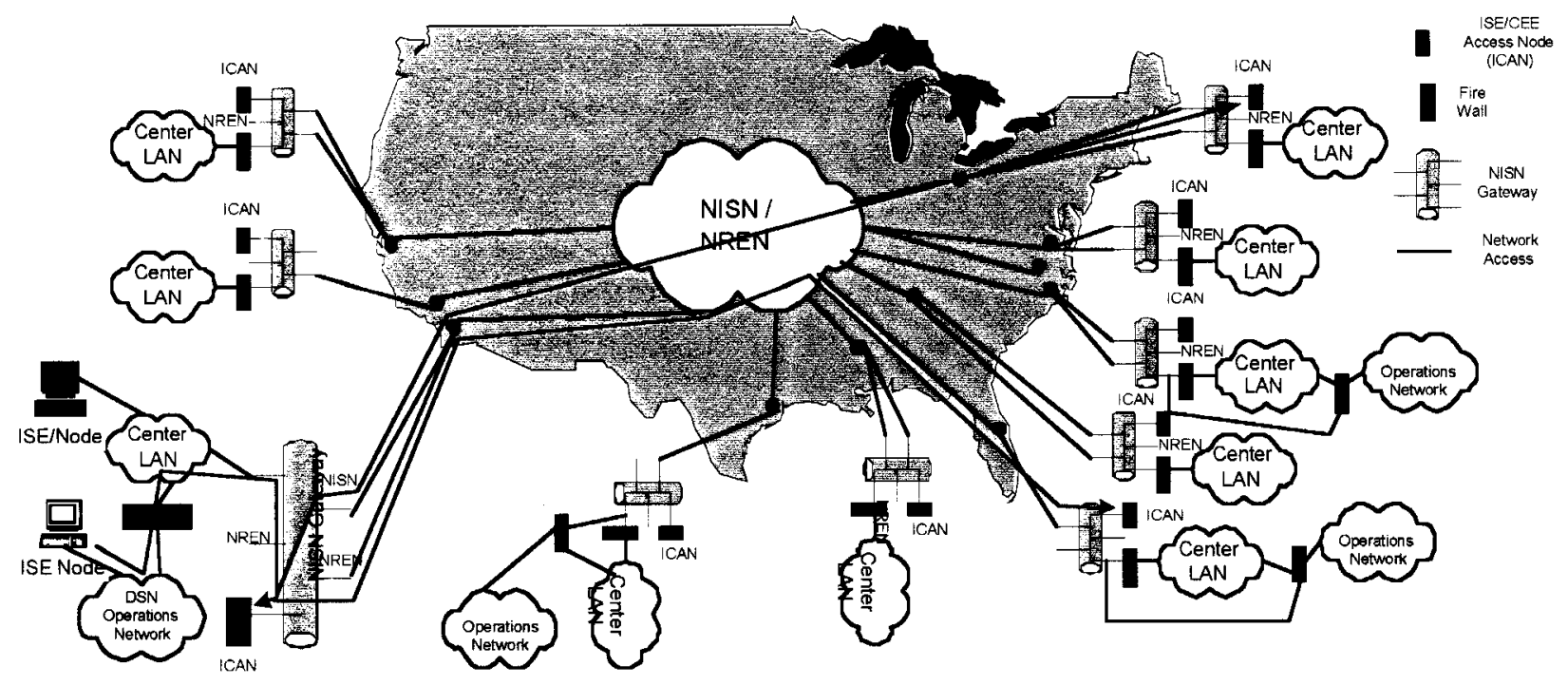

Figure 5 - Collaborative Infrastructure

\section{APPLICATION OF COLLABORATIVE ENGINEERING CAPABILITY}

The integration and application of analysis capabilities is the most challenging aspect of the CEE activities in NASA. The near-term objective is to apply the CEE concept to the design and analysis of several large-scale aerospace systems. An application of the CEE is defined as an integration of people, computer hardware, software, and facilities that enables the development of a specific design and analysis capability. These capabilities focus on a particular design and analysis activity agreed upon by both the CEE element and the sponsoring NASA Program Office. The initial CEE applications have been selected to ensure that a design activity supports each NASA Enterprise (Human Exploration and Development of Space, Space Science, Earth Science, and Aerospace Technology). The current list of applications is as follows:

- Reusable Space Transportation System (RSTS)

Develop and demonstrate an integrated design and analysis environment ( $\mathrm{w} /$ distributed users, applications, and data) to support RSTS design from concept analysis through detailed system design, including mission performance, risk, and lifecycle costs.

- International Space Station

Create a virtual International Space Station "simulator" that will model the ISS vehicle and system performance in any user-selected configuration and environment.

- Space Shuttle

Ability to support/perform virtual assessments (e.g., technical, operational, programmatic) with capability for incorporation of external data for system visualization (e.g., payload integration, Shuttle processing, etc.).

- Advanced Earth Observation System

Mission simulation and simulation-aideddesign of science missions - both Earth and Space Science - starting with the definition of the science measurement and phenomenon, and ending with a visualized simulated validation of the systems designed to take the measurement.

- Integrated Exploration and Science Life cycle simulation of missions to Mars in a realistic martian environment for the 
purpose of mission and system design; project and science planning; and mission operations. The collaborative environment for Mars mission design and analysis will be reusable, shareable, and used for simulating the colonization of Mars by both robotic and human exploration programs.

Each application is being defined and developed by a team of experts from the Enterprise Program Offices (e.g., ISS Program Office), Center engineering organizations, information technology personnel, and ISE advanced engineering team members. This implementation structure ensures that the program offices that will eventually use the final integrated capabilities will also be involved in the application development from the very beginning. Once complete, the integrated capability will allow a team of experts to collaborate on the design and analysis of the Enterprise program missions and systems.

\section{BENEFITS OF COLLABORATIVE ENGINEERING}

The CEE is providing immediate and numerous payoffs and benefits. One major payoff of using the CEE is the potential connection of NASA, other Government Agencies, and Industry in the systems design process. The CEE capabilities developed by NASA can be utilized by all these organizations, and with selected teaming relationships, many organizations will benefit from the technical strengths of the other participants. In a real sense, the CEE could lead to a national collaborative engineering capability that will benefit the United States on a much broader scale and could lead to improved competitiveness of the U.S. aerospace industry. Other substantial benefits are:

- faster aerospace system design cycles - rapid systems development

- less travel - sharing of design tools w/o transferring tools or people

- better cost estimating - cost models and design-to-cost processes integrated

- better access to experts
- quicker response - infrastructure, tools, and processes institutionalized

- less redundancy - collaborative roles defined

- integrated process - transfer of data seamless and well-defined; modeling and simulation well-defined

- tool independence - different tools working to a structured format

- enhanced data perception - improved visualization, sound, etc.

- risk identification leading to reduction and/or mitigation

- improved engineering analysis capabilities well-defined model-driven design process

- improved workforce skills

- improvements in the project earlier in the design process thus reducing costly changes downstream

- improved knowledge retention

- improved capability to rank prospective new projects considering their risk/cost and need

- improved ability to infuse mature technologies into engineering practice

\section{FUTURE COLLABORATIVE ENGINEERING CAPABILITY}

With the development of a NASA Collaborative Engineering Environment being the responsibility of the NASA ISE Program, greater CEE payoffs and benefits are expected as applicable technologies mature and more advanced design and analysis capability is incorporated into the CEE. For example, the plan is for CEE to be continually upgraded with the next generation of tools and methodologies that are developed by other elements of the ISE Program (i.e., Rapid Synthesis and Simulation Tools \{RSST\}, Cost and Risk Management Technologies \{CRMT\}, and Life Cycle Integration and Validation $\{\mathrm{LCIV}\})$. As previously discussed, the application of CEE to large-scale systems designs is planned for implementation over the next 5 years, and as required to accomplish its missions, in the future NASA will add other applications of CEE. The CEE management within the ISE Program is working closely with the NASA Enterprises and program offices to ensure $\mathrm{CEE}$ can provide 
support over the complete system development timeframes. Agreements with the Enterprises will be documented using a mechanism similar to a Memorandum of Agreement (MOAs).

\section{CONCLUSIONS}

Recent advances in computer and communications technologies have enabled the initial implementation of a vision for geographically distributed teaming and fully integrated aerospace system design and analysis capabilities. The extensive and complex NASA missions of the future will require the use of the $\mathrm{CEE}$ capabilities to the greatest extent possible. NASA has recognized the importance of these new capabilities, and priority has been given to implementing CEE so that the Agency can successfully accomplish its missions with efficiency and high technical quality. Substantial progress has been made during the last two years in developing the CEE. The ISE Program, of which CEE is a part, is an approved Agency initiative with a goal of effecting a cultural change that integrates into practice widely distributed science, technology, and engineering teams to rapidly create innovative, affordable aerospace design products. The mission of the CEE element is to implement ISE processes and technologies (e.g., distributed collaborative teaming and life cycle analysis) into engineering practices. In addition, the Agency will work toward the development of new, more advanced design and analysis tools to continually improve the CEE capability and establish NASA as a strong leader in this field. 